\title{
Allen-Like Theory of Time for Tree-Like Structures
}

\author{
S. Durhan ${ }^{\mathrm{a}}$, G. Sciavicco ${ }^{\mathrm{b}}$ \\ ${ }^{a}$ Nesin Mathematics Village, Sirince (Izmir) \\ Turkey,email: salih1@gmail.com \\ ${ }^{b}$ Department of Mathematics, University of Ferrara, \\ Ferrara, Italy, email: guido.sciavicco@unife.it
}

\begin{abstract}
Allen's Interval Algebra is one of the most prominent formalisms in the area of qualitative temporal (and, by extension, spatial) reasoning. However, its applications are naturally restricted to linear flows of time. While there is some recent work focusing on studying relations between intervals (and also between intervals and points) on branching structures, there is no rigourous study of the first-order theory of branching time. In this paper, we approach this problem under a very general definition of time structures, namely, treelike lattices. While Allen's work proved, over thirty years ago, that meets is expressively complete in the linear case, we prove here that, surprisingly, it remains complete for the class of all unbounded tree-like lattices. Such a result cannot be generalized to the case of all tree-like lattices, for which we prove that the smallest complete set of relations has cardinality three. We provide in this paper a sound and complete axiomatic system for both the unbounded and general case, in Allen's style, and we present a systematic study of minimally complete and maximally incomplete sets of relations, again, for both the unbounded and the general case, proving that while in the former case there are precisely four maximally incomplete and eight minimally complete sets, in the latter case these are, respectively, eight and nineteen.
\end{abstract}

Keywords: Representation theorems; branching time; complete and incomplete sets.

\section{Introduction}

Allen's Interval Algebra (IA [1]) is one of the most prominent formalisms in the area of qualitative temporal, and also spatial, reasoning. However, its 
applications are naturally restricted to linear flows of time. Recent work focused on integrating intervals and points to obtain a more general first-order language for linear time [2], and to obtain suitable extensions of Allen's IA for branching time with and without points $[3,4]$. Nonetheless, studying the algebra that emerges from an extended set of relations and its computational complexity is not enough to obtain a clear understanding of the first-order structure that underlies it, and, as witnessed by a famous debate concerning Allen's first-order axiomatization of unbounded interval-based structures (see $[5,6]$ ), this study is not always easy. In this work, we provide a throughout analysis of first-order structures for branching time under minimal hypothesis: we only assume that structures are tree-like lattices, and we study both the unbounded and the general case in terms of a suitable pure firstorder language to express the relations between two intervals on a tree-like lattice in Ligozat's style [7].

In this work, we present, first, (i) a first-order axiomatization of unbounded tree-like lattices, proving that the sole relation meets remains complete, and (ii) a study of minimally complete and maximally incomplete subsets of linear and branching relations for unbounded tree-like lattices. We then focus on the general case, and we prove that meets becomes incomplete when structures are not necessarily unbounded; in this case, we provide, again (iii) a first-order axiomatization of tree-like lattices based on a simple complete set of three relations, and (iv) a study of minimally complete and maximally incomplete subsets of linear and branching relations for tree-like lattices. This problem was left open in [3] (Remark 5), and it turned out to be particularly challenging. Apart from the intrinsic interest in generalizing Allen's results to branching models of time, these results give a concrete answer to the question: When is a given abstract relational structure an interval-structure based on a branching model of time? Being able to answer this question is a key step in constraint satisfaction problems over branching models of time. For example, consider a scheduling problem in which there are two processes which are scheduled to start together and after a certain point they will be running in parallel. Each of these two processes may have their own sub-processes with various scheduling requirements. The whole system then is best modeled in a branching model of time and if one wants to check whether a given schedule is consistent one needs to know, among other things, that the abstract relational algebra described by the schedule is indeed based on a branching model of time. Moreover, intervals in branch- 
ing time are receiving a renewed interested in the field of model checking, historically confined to linear model of time; for example, in [8], the problem of model checking is reformulated in terms of checking interesting properties of computations that make sense on intervals instead of points, and systems give naturally rise to branching structures. Finally, studying the first-order theory of the intervals on branching structures is a necessary preliminary step for the analysis of the complexity of the associated satisfiability (i.e., consistency) problems; these have been partially approached in [3], but are still far from being solved in all aspects.

\subsection{State of the art}

Various representation theorems exist in the literature for languages that include interval relations only on linear time: van Benthem [9], over rationals and with the interval relations during and before, Allen and Hayes [10], for the unbounded case without point intervals and for the relation meets, Ladkin [11], for point-based structures with a quaternary relation that encodes meeting of two intervals, Venema [12], for structures with the relations starts and finishes, Goranko, Montanari, and Sciavicco [13], that generalizes the results for structures with meets and met-by, Bochman [14], for point-interval structures, and Coetzee [15] for dense structure with overlaps and meets. Branching models of time which can be modeled by tree-like structures are of special interest for temporal reasoning at all levels, since they allow for representing non-deterministic aspects of systems, scenarios, and planning tasks. At the modal logic level branching time models have been studied in depth in the recent past. Originated by philosophical logic [16], where branching time logics have been studied for analysis of non-determinism, causality, and action-theoretical concepts, branching time (point-based) logics such as CTL, CTL*, or ATL (see, e.g. $[17,18]$ ) have been proposed as specification languages and, mainly, for model-checking purposes. On the other hand, as far as modal branching time interval-based logics are concerned, the literature is much more scarce; we mention here a future-only branching time version of Propositional Neighborhood Logic studied in [19]. Tree-like structures are a natural choice for modeling temporal aspects of events. For example, in [20] events are defined as closed interval in branching time, and branching structures are exploited to model the different courses that the world might take. The underlying idea is to identify an event with the set of its occurrences in

time. An event may occur in many branches (actually an event is said to occur in a branch if and only if it is completely contained in that branch), 
and a close analysis of this model of time immediately allows one to realize how Allen's relations naturally emerge in this extended context. Also, in the area of automated planning it can be argued that branching time should be chosen as the correct model; as a matter of fact, planning tasks can be modeled as Kripke-like labeled graphs, which naturally unwind to be represented as (potentially unbounded) tree-like structures.

\subsection{Structure of the paper}

In the next section we give the necessary preliminaries. In Section 3 we prove that meets is enough to first-order define any other relation in the context of branching orders, provided that models are unbounded. Section 4, building on the previous result, provides a complete list of minimally complete and maximally incomplete subsets of (linear and branching) relations, again, under the unboundedness assumption. In Section 5 we drop the unboundedness assumption, and show that meets is no longer complete; in this context, we provide a complete (and more complex) first-order axiomatization of the set of all branching models. Finally, in Section 6 we study minimal completeness and maximal incompleteness for subsets of (linear and branching) relations in the class of all branching models.

\section{Preliminaries and Notation}

Let $(\mathcal{T}, \leq)$ be a partial order, whose elements are generally denoted by $a, b, \ldots, x, y, \ldots$, and where $a \| b$ denotes that $a$ and $b$ are incomparable w.r.t. the ordering relation $\leq$. A partial order $(\mathcal{T}, \leq)$, often denoted by $\mathcal{T}$, is a future branching model of time if and only if for all $a, b \in \mathcal{T}$ there is a greatest lower bound of $a$ and $b$ in $\mathcal{T}$, and, if $a \| b$ then there exists no $c \in \mathcal{T}$ such that $c>a$ and $c>b$. Allen's representation theorem [1] works for the class Unb of all unbounded (i.e., such that every point has a successor and a predecessor) linearly ordered sets, and it can be immediately generalized for the class All of all linearly ordered sets [2]. Here, we are interested in:(i) the class TUnb of all unbounded future branching models of time, which immediately generalizes Unb, and (ii) the class TAll of all future branching models of time, which, symmetrically, generalizes All.

An interval in $\mathcal{T}$ is a pair $[a, b]$ where $a<b$, and $[a, b]=\{x \in \mathcal{T}: a \leq x \leq$ $b\}$. Following Allen and Hayes [21], we adopt the so-called strict interpretation by asking that intervals with coincident endpoints are excluded. The 


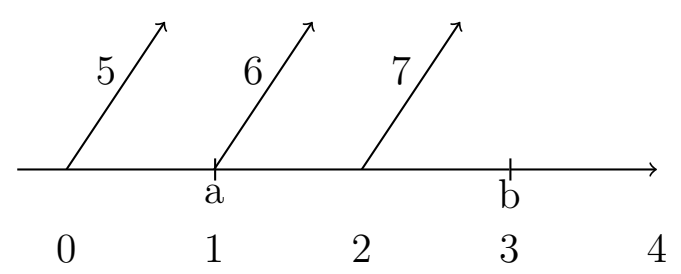

Figure 1: A pictorial representation of the 8 regions arising from an interval on a tree-like lattice.

latter (i.e., the points) can be recovered in an extended point-interval structure and theory, as it has been recently done in the case of linear time [2]. Now, let $\mathcal{I}(\mathcal{T})$ be the set of all intervals $[a, b]$ of $\mathcal{T}$. There are 24 basic relations (including equality) among any two intervals of $\mathcal{T}$, as described, for example, in [3], 13 of which come from Allen's IA over a linear order. As opposed to naming these relations with letters (which come from the initials of verbal description of the relation, such as $m$ to denote that two intervals meet) we shall use a more systematic notation, which is essentially a generalization of the one introduced in [7] and extended in [2]. If $[a, b] \in \mathcal{I}(\mathcal{T})$, then each point $x \in \mathcal{T}$ falls into exactly one of the 8 regions depicted in Fig. 1. In other words, we name with a unique number each set of points that are qualitatively in the same position with respect a given interval $[a, b]$ : for example, region 0 is the set of all points in the domain that are less than (and comparable with) $a$, and region 5 is the set of all points in the domain that are greater than some point $c<a$ are incomparable with $a$. Accordingly, we say that the position of $x$ with respect to $[a, b]$ is $n$ (denoted by $x n[a, b]$ ), where $n \in\{0, \ldots, 7\}$, if $x$ falls into region $n$, as in Tab. 1 . It is clear that in the particular case of $\mathcal{T}$ being a linear order, then we only have the positions $0, \ldots, 4$ (see [7]), which determine whether $x<a, x=a, a<x<b, x=b$ or $b<x$.

In order to describe a relation $\operatorname{Ir} J$ between the intervals $I$ and $J$, we will use the position of the endpoints of $J$ with respect to $I$. In the case when $\mathcal{T}$ is a linear order, the possible positions are $0, \ldots, 4$, and each of Allen's relations are represented by $\operatorname{Inm} J$, where $n, m \in\{0,1,2,3,4\}, n$ is the position of the left endpoint of $J$, and $m$ is the position of the right endpoint of $J$ with respect to $I$. If we take two equal intervals $I=[a, b]$ and $J=[c, d]$, then the position of the left endpoint $J$ with respect to $I$ is 1 and the position of the right endpoint $d$ of $J$ with respect to $I$ is 3 . 


\begin{tabular}{|c|c|c|}
\hline Region & & Semantics \\
\hline$x 0[a, b]$ & $\Leftrightarrow$ & $x<a$ \\
\hline$x 1[a, b]$ & $\Leftrightarrow$ & $x=a$ \\
\hline$x 2[a, b]$ & $\Leftrightarrow$ & $a<x<b$ \\
\hline$x 3[a, b]$ & $\Leftrightarrow$ & $x=b$ \\
\hline$x 4[a, b]$ & $\Leftrightarrow$ & $x>b$ \\
\hline$x 5[a, b]$ & $\Leftrightarrow$ & $x \| a$ \\
\hline$x 6[a, b]$ & $\Leftrightarrow$ & $a<x \wedge(a<y \leq b \rightarrow y \| x)$ \\
\hline$x>[a, b]$ & $\Leftrightarrow$ & $a<x \wedge \exists y(a<y<x \wedge a<y<b)$ \\
\hline
\end{tabular}

Table 1: Notation and semantics of the regions.

$($ meets, $m)[a, b] 34[c, d] \Leftrightarrow b=c$

(before, $b)[a, b] 44[c, d] \Leftrightarrow b<c$

(starts,s) $[c, d] 14[a, b] \Leftrightarrow a=c, d<b$

(finishes,f) $[c, d] 03[a, b] \Leftrightarrow b=d, a<c$

(during, $d)[c, d] 04[a, b] \Leftrightarrow a<c, d<b$

(overlaps,o) $[a, b] 24[c, d] \Leftrightarrow a<c<b<d$

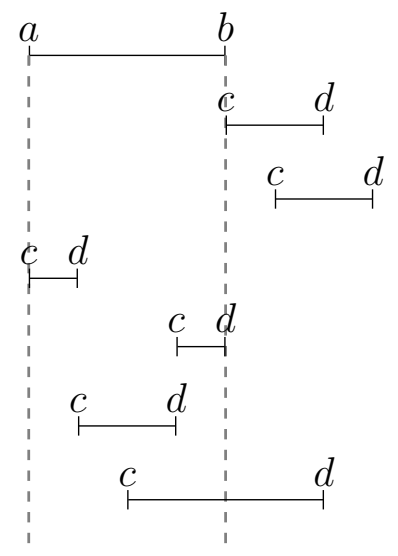

Figure 2: Linear relations, a.k.a. Allen's relations; the equality relation is not depicted.

Therefore equality is represented by $I 13 \mathrm{~J}$. As another example, consider Allen's relation meets, take $I=[a, b], J=[c, d]$ and suppose that $I$ meets $J$. Then $c=b$ and so the position of the left endpoint of $J$ with respect to $I$ is 3. Since $b=c<d$, the position of the right endpoint of $J$ with respect to $I$ is 4. So the relation meets is represented by $I 34 J$. Now, suppose $\mathcal{T}$ is a future branching model of time, and take $I=[a, b], J=[c, d]$, where $I, J \in \mathcal{I}(\mathcal{T})$. Moreover, let $n$ and $m$ be the positions of $c$ and $d$ with respect to the interval $[a, b]$. If both $n, m \in\{0, \ldots, 4\}$, then the pair $n m$ uniquely describes the relation between $I$ and $J$, and we call these linear relations; they are (the 


$$
\begin{aligned}
& {[b, c] 06[a, e]} \\
& {[b, d] 07[a, g]} \\
& {[c, d] 55[e, f]} \\
& {[b, c] 66[e, f]} \\
& {[b, c] 16[b, e]} \\
& {[b, d] 17[b, g]} \\
& {[b, d] 77[g, h]}
\end{aligned}
$$



Figure 3: Determined non-linear relations.

well-known) Allen's relations, and they are depicted, along with the notation we use for them, in Fig. 2. Otherwise, the relation is called non-linear, and these require more careful consideration. Suppose the intervals $I, J$ stand in some non-linear relation. For the cases described in Fig. 3, called determined non-linear relations, again the pair $(n, m)$ uniquely determines the relation between $I=[a, b]$ and $J=[c, d]$, which we denote by $I n m J$. On the other hand, when $n m$ is equal to 05 or 27 there are (only) two possible relations between $I$ and $J$. To resolve this issue we introduce a specific notation for these undetermined non-linear relations in order to distinguish the possible cases, namely we use $I n m J$ or $I n m^{e} J$. Notice that in [2], an ontology which covers both intervals and points is considered, and, therefore, on top of relations among intervals there are interval-point relations and point-point relations. To distinguish relations among intervals, the notation $n m_{i i}$ is used; we avoid this use of subscripts in our notation since we only consider relations among intervals. The semantics of both determined and undetermined relations can be specified as follows:

$$
\begin{aligned}
& {[a, b] 06[c, d] \quad \Leftrightarrow \quad c<a<d \wedge \forall x(a<x \leq d \rightarrow x \| b)} \\
& {[a, b] 07[c, d] \quad \Leftrightarrow \quad c<a<d \wedge d \| b \wedge \exists x(a, c<x<b, d)} \\
& {[a, b] 16[c, d] \quad \Leftrightarrow \quad c=a \wedge \forall x(c<x \leq d \rightarrow x \| b)} \\
& {[a, b] 17[c, d] \quad \Leftrightarrow \quad c=a \wedge d \| b \wedge \exists x(a, c<x<b, d)} \\
& {[a, b] 55[c, d] \quad \Leftrightarrow \quad c \| a} \\
& {[a, b] 66[c, d] \quad \Leftrightarrow \quad a<c \wedge \forall x(a<x \leq b \rightarrow x \| c)} \\
& {[a, b] 77[c, d] \quad \Leftrightarrow \quad a<c \wedge b \| c \wedge \exists x(a<x<b, c)} \\
& {[a, b] 05[c, d] \quad \Leftrightarrow \quad c<a \wedge \forall x(c<x \leq d \rightarrow x \| a)} \\
& {[a, b] 05^{e}[c, d] \quad \Leftrightarrow \quad c<a \wedge a \| d \wedge \exists x(c<x<d \wedge x<a)} \\
& {[a, b] 27[c, d] \quad \Leftrightarrow \quad a<c<b \wedge \forall x(c<x \leq d \rightarrow x \| b)} \\
& {[a, b] 27^{e}[c, d] \quad \Leftrightarrow \quad a<c<b \wedge d \| b \wedge \exists x(a, c<x<b, d)}
\end{aligned}
$$


For any relation $r$ between two intervals, its inverse relation, denoted by $\bar{r}$, is the relation

$$
I \bar{r} J \leftrightarrow J r I
$$

In the cases of linear relations, the inverses of $00,01,02,23,22,12$ are 44, 34, 24, $03,04,14$, respectively. Note that the linear relation 13 is the equality of intervals, which will be denoted $=$, and its inverse is itself. Moreover, from the above description, it is easy to see that the inverses of $05,05^{e}, 27,27^{e}$ are $66,77,06,07$, respectively, and the inverses of 16,17 and 55 are themselves. Since we deal exclusively with first-order properties, we can omit the relations that may be defined simply as the inverse of another relation. Hence we define the interval language over future branching models of time, denoted by $\mathcal{L}_{B I}$, as $\mathcal{L}_{L I} \cup \mathcal{D}$, where $\mathcal{L}_{L I}$ is the interval language over linear models of time (i.e., Allen's language, which includes the six basic linear relations together with equality), and $\mathcal{D}$ is the set of all and only determined non-linear relations. Given a future branching model of time $\mathcal{T}$, the structure $\mathcal{I}(\mathcal{T})$ will be considered as a $\mathcal{L}_{B I}$-structure where the relations $n m$ are interpreted as described above. Therefore, for every relation $n m \in \mathcal{L}_{B I}$, we have that $[a, b] n m[c, d]$ if and only if $n$ is the position of $c$ and $m$ is the position of $d$ with respect to $[a, b]$ (notice that our choice w.r.t. the relations that are included in the language allows us to avoid the problem that arises from undetermined non-linear relations).

Definition 1. Given a class of partial (resp., linear) orders $\mathcal{C}$, the theory of future branching time intervals (resp., of linear time intervals) over $\mathcal{C}$ is the set of all $\mathcal{L}_{B I}$-sentences (resp., $\mathcal{L}_{L I}$-sentences) which hold in every $\mathcal{I}(\mathcal{T})$ (resp., every $\mathcal{I}(\mathcal{T})$ where $\mathcal{T}$ is a linear order) that belong to the class $\mathcal{C}$, and it is denoted by $\Sigma_{B I}^{\mathcal{C}}$ (resp., by $\Sigma_{L I}^{\mathcal{C}}$ ). A future branching (resp., linear) interval structure is any $\mathcal{L}_{B I}^{\mathcal{C}}$-structure (resp., $\mathcal{L}_{L I}^{\mathcal{C}}$-structure) $\mathcal{I}$ such that $\mathcal{I}=\Sigma_{B I}^{\mathcal{C}}$ (resp., $\left.\mathcal{I}=\Sigma_{L I}^{\mathcal{C}}\right)$. The theory $\Sigma_{B I}^{\mathrm{TAll}}$ (resp., $\Sigma_{L I}^{\text {All }}$ ) is simply denoted by $\Sigma_{B I}$ (resp., $\left.\Sigma_{L I}\right)$.

Given a language $\mathcal{L} \in\left\{\mathcal{L}_{L I}, \mathcal{L}_{B I}\right\}$, a subset $S \subseteq \mathcal{L}$, and a relation $r \in \mathcal{L}$, we say that $S$ defines $r$ over $\mathrm{C}$, denoted by $S_{\mathcal{C}} \rightarrow r$, if it is the case that $\Sigma_{L}^{\mathcal{C}} \models(\operatorname{Ir} J) \leftrightarrow \varphi(I, J)$, for some $\mathcal{S}$-formula $\varphi$. Notice that definability is preserved from a class $\mathcal{C}$ to a smaller one $\mathcal{C}^{\prime}$. The set $S$ is $\mathcal{L}$-complete over $\mathcal{C}$ if and only if $S \rightarrow_{\mathcal{C}} r$ for all $r \in \mathcal{L}$; minimally $\mathcal{L}$-complete over $\mathcal{C}$, denoted $\operatorname{mcs}(\mathcal{C})$ (resp., maximally $\mathcal{L}$-incomplete over $\mathrm{C}$, denoted $\operatorname{MIS}(\mathcal{C})$ ) if and only if it is $\mathcal{L}$-complete (resp., $\mathcal{L}$-incomplete) over $\mathcal{C}$, and, every proper subset (resp., 
every strict superset) of $S$ is $\mathcal{L}$-incomplete (resp., $\mathcal{L}$-complete) over the same class. For example, Allen and Hayes [10] proved that meets (i.e., 34 in our notation) is $\mathcal{L}_{L I}$-complete over Unb, and, as we have recalled, it immediately generalizes to prove that 34 is also $\mathcal{L}_{L I}$-complete over All. While proving $\mathcal{L}$-completeness of a set of relations requires (a set of) defining first-order formulas, proving incompleteness is obtained by means of a bisimulation argument, as follows.

Definition 2. Given a set of relations $S$, and two structures $\mathcal{I}(\mathcal{T})$ and $\mathcal{I}\left(\mathcal{T}^{\prime}\right)$ based on future branching models of time $(\mathcal{T}, \leq)$ and $\left(\mathcal{T}^{\prime}, \leq\right)$, a binary relation $f \subseteq \mathcal{I}(\mathcal{T}) \times \mathcal{I}\left(\mathcal{T}^{\prime}\right)$ is called a surjective $S$-truth preserving relation if and only if:

(i) $f$ respects the relations in $S$, i.e., if $\left(I, I^{\prime}\right),\left(J, J^{\prime}\right) \in f$, then $r(I, J)$ if and only if $r\left(I^{\prime}, J^{\prime}\right)$ for every relation $r \in S$;

(ii) $f$ is total and surjective.

If $f$ is a surjective $S$-truth preserving relation, we say that $f$ breaks $r \notin S$ if and only if there are $\left(I, I^{\prime}\right),\left(J, J^{\prime}\right) \in f$ such that $r(I, J)$ but $\neg r\left(I^{\prime}, J^{\prime}\right)$.

As one would expect, surjective $S$-truth preserving relations preserve the truth of all first-order formulas in signature $S$, as proven by a straightforward generalization of the classical result on the preservation of truth under isomorphism between first-order structures.

Theorem 1. If $f$ is a surjective $S$-truth preserving relation between $\mathcal{I}(\mathcal{T})$ and $\mathcal{I}\left(\mathcal{T}^{\prime}\right)$, and $I_{1}, \ldots, I_{l}, I_{1}^{\prime}, \ldots, I_{l}^{\prime}$ are intervals of $\mathcal{I}(\mathcal{T})$ and $\mathcal{I}\left(\mathcal{T}^{\prime}\right)$, respectively, such that $\left(I_{j}, I_{j}^{\prime}\right) \in f$ for $1 \leq j \leq l$, then for every first-order formula $\varphi\left(x_{1}, \ldots, x_{l}\right)$ in the signature $S$, with free variables $x_{1}, \ldots x_{l}$, we have that

$$
\mathcal{I}(\mathcal{T}) \models \varphi\left(I_{1}, \ldots, I_{l}\right) \text { if and only if } \mathcal{I}\left(\mathcal{T}^{\prime}\right) \models \varphi\left(I_{1}^{\prime}, \ldots, I_{l}^{\prime}\right) \text {. }
$$

Therefore, in order to show that a certain subset of relations $S$ is $\mathcal{L}$-incomplete, it suffices to provide a surjective $S$-truth preserving relation $f$ between two structures $\mathcal{I}(\mathcal{T})$ and $\mathcal{I}\left(\mathcal{T}^{\prime}\right)$ such that $f$ breaks at least one relation $r \in \mathcal{L} \backslash S$. When $\mathcal{L}$-incompleteness is restricted to a certain class of structures $\mathcal{C}$, then both structures $\mathcal{I}(\mathcal{T})$ and $\mathcal{I}\left(\mathcal{T}^{\prime}\right)$ must be based on frames $\mathcal{T}, \mathcal{T}^{\prime} \in \mathcal{C}$. 


\section{Completeness over TUnb}

In this section we prove that, perhaps surprisingly, Allen's relation 34 is $\mathcal{L}_{B I}$ complete over TUnb. The completeness of 34 in the linear unbounded case, recalled in the previous sections, and studied also in [22], does not necessarily generalize to TUnb, as the relative definitions might change. Notice also that we state the result for the class TUnb while, in actuality, they hold for the super-class of TUnb that includes tree-lattices that are not necessarily unbounded to the left (only to the right). Proofs are stated in this slightly more general setting; the axioms themselves are identical for the two cases with the exception of the last one, which instead requires a slight change.

Lemma 1. For each $r \in \mathcal{L}_{L I},\{34\} \rightarrow_{\text {TUnb }} r$.

Proof. To prove this result we can limit ourselves to presenting the set of all defining formulas for each linear relation in terms of 34. A possible solution is:

$$
\begin{aligned}
I=J & \leftrightarrow \forall K(K 34 I \leftrightarrow K 34 J) \wedge \forall K(I 34 K \leftrightarrow J 34 K), \\
I 44 J & \leftrightarrow \exists K(I 34 K \wedge K 34 J), \\
I 14 J & \leftrightarrow \forall K(K 34 I \leftrightarrow K 34 J) \wedge \exists K \forall L((I 34 K) \wedge(K 34 L \leftrightarrow J 34 L)), \\
I 03 J & \leftrightarrow \forall K(I 34 K \leftrightarrow J 34 K) \wedge \exists K(K 34 I \wedge K 14 J), \\
I 04 J & \leftrightarrow \exists K, L(K 34 I \wedge K 14 J \wedge I 34 L \wedge L 03 J), \\
I 24 J & \leftrightarrow \exists K, L(K 14 I \wedge K 34 J \wedge I 34 L \wedge L 03 J) .
\end{aligned}
$$

The above definitions are relatively straightforward, and can be easily proved correct.

So far we have derived all the linear relations in $\mathcal{L}_{B I}$ from 34 ; now we can use all of them to derive the remaining (non-linear) relations. To this end, we introduce a new relation that holds when $I$ and $J$ do not satisfy any linear relations, by imposing that:

$$
I 6 r J \leftrightarrow\left(\bigwedge_{r \in \mathcal{L}_{L I}} \neg(\operatorname{Ir} J)\right) \wedge\left(\bigwedge_{r \in \mathcal{L}_{L I}} \neg(\operatorname{Jr} I)\right) .
$$

We denote the complement of the relation $6 r$ by $l i$, which of course contains the pairs of intervals which are related through some linear interval relation. Note that the following observation about $6 r$ holds in the general context of TAll. 
Lemma 2. If $I=[a, b]$ and $J=[c, d]$, then $\Sigma_{B I}^{\mathrm{TAll}} \models I b r J$ if and only if $b \| d$.

Proof. Suppose that $I 6 r J$ holds; we want to show that $b \| d$. Since $I$ and $J$ do not satisfy any linear relation, we have that either $a\|c, b\| c, a \| d$ or $b \| d$. If $a \| c$, since $a<b$ and $c<d$, we get $b \| d$ because $\mathcal{T}$ is future branching. If $b \| c$, since $c<d$, we get, again, $b \| d$. Likewise, if $a \| d, a<b$ leads to $b \| d$. As for the other direction, it is clear that if $b \| d$ then $I$ and $J$ do not satisfy any linear relation.

Lemma 3. For each $r \in \mathcal{L}_{B I} \backslash \mathcal{L}_{L I},\{34\} \rightarrow_{\text {TUnb }} r$.

Proof. As in Lemma 1, we can limit ourselves to listing all necessary definitions. A possible solutions is:

$$
\begin{aligned}
I 17 J & \leftrightarrow I 6 r J \wedge \forall K(K 34 I \leftrightarrow K 34 J) \wedge \exists K(K 14 I \wedge K 14 J), \\
I 16 J & \leftrightarrow I 6 r J \wedge \forall K(K 34 I \leftrightarrow K 34 J) \wedge \neg(I 17 J), \\
I 55 J & \leftrightarrow \exists K, L(K 34 I \wedge L 34 J \wedge K 16 L), \\
I 66 J & \leftrightarrow \exists K(K 16 I \wedge K 34 J), \\
I 77 J & \leftrightarrow \exists K(K 17 I \wedge K 34 J), \\
I 06 J & \leftrightarrow \exists K(K 16 I \wedge K 03 J), \\
I 07 J & \leftrightarrow \exists K(K 17 I \wedge K 03 J) .
\end{aligned}
$$

Once again, all the above are relatively straightforward, and it is easily seen that they are correct under the assumption of unboundedness.

Theorem 2. The set $\{34\}$ is minimally $L_{B I}$-complete on TUnb.

Now we will provide an axiomatization for $\Sigma_{B I}^{T U n b}$. As we have mentioned, the provided axiomatization generalizes Allen's set of first-order conditions for an abstract structure to be interpreted as an unbounded branching model of time (as opposed to an unbounded linear order). First, let us introduce two new relations, defined using only 34, which make the exposition and the axioms clearer.

$$
I \sim J \leftrightarrow \forall K(K 34 I \leftrightarrow K 34 J)
$$

The relation $\sim$ is an equivalence by definition, regardless of the semantics of 34; in the semantics of interval structures, $I \sim J$ if and only if $I$ and $J$ have the same starting point, and we can easily use $\sim$ to define a partial ordering on the starting points of intervals:

$$
I<J \leftrightarrow \exists K(K \sim I \wedge K 34 J) .
$$


(1) $\forall I, J((\exists K(K 34 I \wedge K 34 J)) \rightarrow \forall K(K 34 I \rightarrow K 34 J))$

(2) $\forall I, J((\exists K(I 34 K \wedge J 34 K)) \rightarrow \forall K(I 34 K \rightarrow J 34 K))$

(3) $\forall I(\neg I<I) \wedge \forall I, J(I<J \rightarrow J \nless I) \wedge \forall I, J, K((I<J \wedge J<K) \rightarrow I<K))$

(4) $\forall I, J \exists K(K \leq I \wedge K \leq J \wedge \forall L((L \leq I \wedge L \leq J) \rightarrow L \leq K))$

(5) $\forall I, J, K((I|| J \wedge I \not J) \rightarrow \neg(I<K \wedge J<K))$

(6) $\forall I \exists K(I 34 K) \wedge \forall I \exists K(K 34 I)$

Table 2: Axiomatization of TUnb in the language $L_{B I}$ (where the underlined conjunct is added - it must be eliminated to axiomatize the super-class of TUnb that includes possibly left-bounded tree-like lattices).

Let $\leq$ be a shorthand for the formula $(I<J \vee I=J)$, and let $I \| J$ be a shorthand for $(I \nless J \wedge J \nless I)$.

Lemma 4. The relation $<$ induces a well-defined relation on the set of equivalence classes of $\sim$. That is, if $I<J, I \sim I^{\prime}$ and $J \sim J^{\prime}$ then $I^{\prime}<J^{\prime}$.

Proof. Suppose $I<J, I \sim I^{\prime}$ and $J \sim J^{\prime}$. Let $K$ be such that $K \sim I$ and $K 34 J$. Since $\sim$ is an equivalence relation, $K \sim I^{\prime}$ and since $J \sim J^{\prime}$ we have $K 34 J^{\prime}$. Therefore $I^{\prime}<J^{\prime}$.

Again, note that the above lemma is independent of the semantics of the relation 34 .

The first two axioms in Tab. 2 are based on the intuition that every interval should have a unique starting point and a unique ending point. Axiom (3) expresses the idea that $<$ is a partial ordering. Also, we want to ensure that the partial ordering induced by $<$ on the equivalence classes of $\sim$ has greatest lower bounds and that incomparable elements do not become comparable in the future (so it is future branching), and to this end we use Axiom (4) and (5). To express unboundedness we use Axiom (6). The second conjunct of Axiom (6), underlined, can be added to obtain a representation theorem for TUnb, or eliminated to obtain a representation theorem for the superclass of TUnb of tree-like lattices unbounded to the right and not necessarily unbounded to the left.

Theorem 3. Let $\mathcal{I}$ be a $\{34\}$-structure satisfying Axioms (1) to (6) (including the last conjunct) in Tab. 2. Then, there is an unbounded future branching 
model of time $\mathcal{T}$, definably interpretable in $\mathcal{I}$, such that $\mathcal{I}$ is isomorphic to $\mathcal{I}(\mathcal{T})$.

Proof. Let $\mathcal{T}=\mathcal{I} / \sim$, the set of equivalence classes of $\sim$ over $\mathcal{I}$. By Lemma $4,<$ induces a well-defined relation on $\mathcal{T}$ which we also denote by $<$. Note that $(\mathcal{T},<)$ is definably interpretable in $\mathcal{I}$, and, moreover, by Axiom (3), (4), (5), and (6), it is an unbounded future branching model of time. Now, for $\mathcal{L}=\{34\}$, we consider the set of intervals over $\mathcal{T}$ as a $\mathcal{L}$-structure where 34 is interpreted in the natural way, and define $f: \mathcal{I} \rightarrow \mathcal{I}(\mathcal{T})$ as:

$$
f(I) \mapsto[I / \sim, J / \sim]
$$

where $J \in \mathcal{I}$ with $I 34 J$. Note that $J / \sim$ is independent of the choice of $J$. If $J^{\prime}$ is any other interval such that $I 34 J^{\prime}$, then by (1) we have $J \sim J^{\prime}$; moreover, the existence of such $J$ is guaranteed by Axiom (6). We claim that $f$ is an isomorphism of $\mathcal{L}$-structures. Suppose, first, that $f(I)=f(J)$; then $I / \sim=J / \sim$, and there is $K$ such that $I 34 K$ and $J 34 K$. Using Axiom (1) and (2) it is easy to see that $I=J$. Note that $=$ is interpreted in $\mathcal{I}$ by the definition provided in Lemma 1 . So $f$ is one-to-one. Now, let $[I / \sim, J / \sim] \in \mathcal{I}(\mathcal{T})$. Then, $I / \sim<J / \sim$, and, by the definition of $<$, there is $K \in \mathcal{I}$ such that $K \sim I$ and $K 34 J$. Therefore $f(K)=[I / \sim, J / \sim]$, and, so, $f$ is onto. Finally, we need to show that $f$ respects the relation 34. To this end, take $I, J \in \mathcal{I}$ such that $I 34 J$ and $f(I)=[I / \sim, K / \sim]$, $f(J)=[J / \sim, L / \sim]$. Then, $K$ is any interval such that $I 34 K$. Since we also have $I 34 J, K \sim J$ by (1), and, hence, $f(I) 34 f(J)$ as we wanted.

Theorem 4 (Representation Theorem). $\Sigma_{B I}^{\text {TUnb }}$ is axiomatized by Axioms (1) to (6) (including the last conjunct) in Tab. 2, together with the definitions of all relations in $\mathcal{L}_{B I}$ from 34.

Proof. It is clear that if $\mathcal{T}$ is an unbounded future branching model of time then (1) to (6) hold in $\mathcal{I}(\mathcal{T})$. Suppose that $\mathcal{I}$ is an $\mathcal{L}_{B I}$ structure satisfying (1) to $(6)$, where each relation in $\mathcal{L}_{B I}$ is interpreted according to the definitions provided in Lemma 1 and 3. Then, by Theorem 3, $\mathcal{I}$ is isomorphic to an interval structure $\mathcal{I}(\mathcal{T})$ and therefore $\mathcal{I} \models \Sigma_{B I}^{\text {TUnb }}$.

\section{Expressive Power over TUnb}

To complete our analysis of the class TUnb, we ask ourselves which subsets of $\mathcal{L}_{B I}$ are minimally complete and which are maximally incomplete, 


\begin{tabular}{|l|l|}
\hline $\operatorname{MIS}\left(\mathcal{L}_{B I}\right) \mathrm{s}$ & $\operatorname{mcs}\left(\mathcal{L}_{B I}\right) \mathrm{s}$ \\
\hline$\{03,=\} \cup\left(\mathcal{L}_{B I} \backslash \mathcal{L}_{L I}\right)$ & $\{34\}$ \\
$\{14,=\} \cup\left(\mathcal{L}_{B I} \backslash \mathcal{L}_{L I}\right)$ & $\{14,24\}$ \\
$\{04,=\} \cup\left(\mathcal{L}_{B I} \backslash \mathcal{L}_{L I}\right)$ & $\{03,24\}$ \\
$\{24,=\} \cup\left(\mathcal{L}_{B I} \backslash \mathcal{L}_{L I}\right)$ & $\{14,04\}$ \\
& $\{03,04\}$ \\
& $\{14,03\}$ \\
& $\{04,24\}$ \\
& $\{44\}$ \\
\hline
\end{tabular}

Table 3: Maximally $\mathcal{L}_{B I}$-incomplete (left-hand side) and minimally $\mathcal{L}_{B I}$-complete sets (right-hand side) over TUnb.

borrowing (and extending) some constructions from [22] to this end, keeping in mind that even for linear interval relations definability equations over future branching models of time may be different from the corresponding definitions over linear models of time. The main reason for this difference is that over branching models time the negation of $b \leq c$ is not $c<b$ anymore, and this obvious difference makes definability relations more difficult to devise. In the following, we shall make extensive use of Theorem 2, which implies that if 34 is definable from $S$ then $S$ is complete.

Lemma 5. Each set listed in the right-hand side column of Tab. 3 is $\mathcal{L}_{B I^{-}}$ complete over TUnb.

Proof. The completeness of 34 is proved in Theorem 2, and its minimality is obvious; in the rest of the proof, we list a set of equations for the remaining sets. We first consider the case $S=\{14,24\}$; with the aim to define the linear relation 34 (and, therefore, prove the completeness of the considered set), we define the weaker relation $34 \cup 44$, which holds between $[a, b]$ and $[c, d]$ if and only if $b \leq c$, instead, as:

$$
I 34 \cup 44 J \leftrightarrow \exists K(I 14 K \wedge \forall L(J 14 L \rightarrow K 24 L)) \wedge \wedge \forall K(J 14 K \rightarrow \neg I 24 K) .
$$

Then, from the above relation it is immediate to obtain 34 :

$$
I 34 J \leftrightarrow I 34 \cup 44 J \wedge \forall K(I 34 \cup 44 K \rightarrow \neg(K 34 \cup 44 J) .
$$


The case $S=\{24,03\}$ is very similar to the previous one:

$$
\begin{aligned}
I 34 \cup 44 J \leftrightarrow & \exists K(J 03 K \wedge \forall L(I 03 L \rightarrow L 24 K)) \wedge \\
& \forall K(I 03 K \rightarrow \neg K 24 J),
\end{aligned}
$$

whose correctness is immediate. As for the set $S=\{14,04\}$, we prove its completeness by defining 24 in it, and, then, resorting to the previous results:

$$
I l i J \leftrightarrow \exists K(I 04 K \wedge J 04 K) .
$$

By imposing $I$ li $J$ we make sure that $I$ and $J$ are related by some $r \in \mathcal{L}_{L I}$; then, the unboundedness of the considered models, as well as the fact that they are future branching, play an essential role in this definition:

$$
\begin{aligned}
I 24 J \leftrightarrow & \exists K\left(I 14 K \wedge \neg(J 04 K) \wedge \exists K^{\prime}\left(K^{\prime} 04 K \wedge K^{\prime} 14 J\right)\right) \wedge \\
& \exists K\left(K 14 J \wedge \forall K^{\prime}\left(I 04 K^{\prime} \rightarrow K 04 K^{\prime}\right)\right) \wedge \\
& \text { I liJ. }
\end{aligned}
$$

In a similar, but simpler, way we can prove that $S=\{14,03\}$ is complete. Indeed, the following formula defines 34 :

$$
I 34 \cup 44 J \leftrightarrow \exists K(I 14 K \wedge J 03 K) \wedge \neg \exists K(K 03 I \wedge K 14 J) .
$$

It is immediate to see that the above definition is correct. We now have to consider the set $S=\{24,04\}$, and we do so by means of the following definition:

$$
\begin{aligned}
I 34 \cup 44 J \leftrightarrow & I f i J \wedge \exists K, K^{\prime}\left(I 04 K \wedge J 04 K^{\prime} \wedge K 24 K^{\prime}\right) \wedge \\
& \exists K(I 04 K \wedge \neg(J 04 K)) \wedge \exists K(J 04 K \wedge \neg(I 04 K)) \wedge \\
& \neg(I 24 J) \wedge \neg(J 24 I) \wedge \neg(I 04 J) \wedge \neg(J 04 I) .
\end{aligned}
$$

To complete this proof we have to show that $\{44\}$ defines 34 . We do so by proving that $\{44\}$ defines both 14 and 03 , and, then, resorting to the completeness of $\{14,03\}$ :

$$
\begin{aligned}
I 14 J \leftrightarrow & \forall K(K 44 I \leftrightarrow K 44 J) \wedge \exists K(I 44 K \wedge \neg J 44 K) \wedge \\
& \forall K(J 44 K \rightarrow I 44 K) \\
I 03 J \leftrightarrow & \forall K(J 44 K \leftrightarrow I 44 K) \wedge \exists K(K 44 I \wedge \neg K 44 J) \wedge \\
& \forall K(K 44 J \rightarrow K 44 I) .
\end{aligned}
$$


Lemma 6. Each set listed in the left-hand side column of Tab. 3 is $\mathcal{L}_{B I^{-}}$ incomplete over TUnb.

Proof. Let $S=\{14,=\} \cup\left(\mathcal{L}_{B I} \backslash \mathcal{L}_{L I}\right)$. Borrowing the construction from [22], we take $\mathcal{T}=\mathbb{Q}$ with the usual ordering, and $f: \mathcal{I}(\mathcal{T}) \rightarrow \mathcal{I}(\mathcal{T})$ that sends every interval $[a, b]$ to $[a, a+2 \cdot|b-a|]$. In other words, the image of any interval $[a, b]$ under $f$ has the same beginning point, but double the length of $[a, b]$. Notice that we are allowed to consider linear structures, as they are simply degenerate branching structures. We claim that $f$ preserves every relation in $S$ : first, observe that it is a bijection, so equality is preserved; then, it is easy to see that every non-linear relation is also preserved, because the structure is linear; finally, we have that $[a, b] 14[c, d]$ if and only if $a=c$ and $b<d$, that is, if and only if $a=c$ and $a=c$ and $a+2 \cdot|b-a|<c+2 \cdot|d-c|$, which happens if and only if the image under $f$ of $[a, b]$ is in the relation 14 with the image under $f$ of $[c, d]$. But, for example, 44 is broken by $f$ : indeed, $[0,1] 44[2,3]$ but $[0,2] 44[2,4]$. A similar, but symmetric, argument, works to prove that $S=\{03,=\} \cup\left(\mathcal{L}_{B I} \backslash \mathcal{L}_{L I}\right)$ is also incomplete. Suppose, now, that $S=\{04,=\} \cup\left(\mathcal{L}_{B I} \backslash \mathcal{L}_{L I}\right)$ Again, we take $\mathcal{T}=\mathbb{Q}$ with the usual ordering, and $f: \mathcal{I}(\mathcal{T}) \rightarrow \mathcal{I}(\mathcal{T})$ that sends every interval $[a, b]$ to $[-b,-a]$. Clearly, the relation 04 is preserved; since the structure is linear, as before, every non-linear relation is preserved as well, and since $f$ is a bijection, equality is preserved as well. But every linear relation other than 04 is broken, proving that $S$ is incomplete. As for $S=\{24,=\} \cup\left(\mathcal{L}_{B I} \backslash \mathcal{L}_{L I}\right)$, we take $\mathcal{T}=\mathbb{Z}$, and $f: \mathcal{I}(\mathcal{T}) \rightarrow \mathcal{I}(\mathcal{T})$ that sends every interval $[a, a+1]$ to $[a+1, a+2]$, and every other interval to itself. As before, non-linear relations are trivially preserved, as it is equality; since only unitary intervals are affected by $f$, the relation 24 must be preserved as well. Nonetheless, every linear relation other than 24 is broken, proving that $S$ is incomplete.

Theorem 5. Tab. 3 contains all minimally $\mathcal{L}_{B I}$-complete and maximally $\mathcal{L}_{B I}$-incomplete subsets of $\mathcal{L}_{B I}$ over TUnb.

Proof. Observe that in Tab. 3 every subset of a complete set is contained in an incomplete set, and every superset of an incomplete set contains a complete set. Moreover, it is easy to see that each given subset of $\mathcal{L}_{B I}$ is either (not necessarily strictly) contained in one of those listed in the lefthand side column or it (not necessarily strictly) contains one of those listed in the right-hand side column. Therefore, the left-hand side column of Table 3 
is the list of all maximally incomplete sets and the rightmost column is the list of all minimally complete sets over TUnb.

\section{Completeness over TAll}

In this section, we consider the class TAll of all tree-like lattices. In sharp contrast with Allen's original result, and our results of the previous sections, it turns out that the set $\{34\}$ is no longer complete in this case. Moreover, its incompleteness, paired with the presence of irregular tree-like lattices in the class (such as those lattices with an infinite number of unbounded branches and an infinite number of bounded ones) make the problem of devising an axiomatization of the class TAll particularly challenging. First, we prove that $\{34\}$ is incomplete for the class TAll.

Lemma 7. The sets $\{34,03\},\{=, 03\}$ and $\{=, 34\}$ are incomplete over TAll.

Proof. Consider, first, the case $\{34,03\}$. Let $\mathcal{T}=\{a, b, c\}$ ordered by $a<b$, $a<c$ and $b \| c$, and let $f: \mathcal{I}(\mathcal{T}) \rightarrow \mathcal{I}(\mathcal{T})$ be the map which sends both $[a, b]$ and $[a, c]$ to $[a, b]$. Since there are no intervals over $\mathcal{T}$ which are related via 34 nor 03 , both these relations are preserved. Clearly, $=$ is broken, and, so, $\{34,03\}$ is not complete. Next, consider the case $\{=, 03\}$. Let $\mathcal{T}=\{a, b, c, d\}$ ordered by $a<b<c, a<d$ and $d \| b$, and let $f: \mathcal{I}(\mathcal{T}) \rightarrow \mathcal{I}(\mathcal{T})$ be the map which sends $[a, b]$ to $[a, d],[a, d]$ to $[a, b]$ and keeps all other intervals fixed. Since $f$ is one-to-one it preserves $=$, and the only intervals related through 03 are $[a, c]$ and $[b, c]$, which are sent to themselves. So $f$ preservers 03 as well. However, $[a, b] 34[b, c]$ but $\neg([a, d] 34[b, c])$, so $\{=, 03\}$ is not complete. Finally, consider the case $\{=, 34\}$. Let $\mathcal{T}=\{a, b, c, d\}$ ordered by $a<b<c, a<d$ and $d \| b$. Let $f: \mathcal{I}(\mathcal{T}) \rightarrow \mathcal{I}(\mathcal{T})$ be the map which sends $[a, d]$ to $[a, c],[a, c]$ to $[a, d]$, and keeps all other intervals fixed. Since $f$ is one-to-one it preserves $=$, and the only intervals related through 34 are $[a, b]$ and $[b, c]$, which are sent to themselves. So, $f$ preserves 34 as well. However, $[b, c] 03[a, c]$ but $\neg([b, c] 03[a, d])$. So $\{=, 34\}$ is not complete.

By suitably combining the above incomplete sets, we can easily devise a first complete set in the case of TAll.

Lemma 8. For each $r \in \mathcal{L}_{L I},\{=, 34,03\} \rightarrow_{\text {TAll }} r$. 




Table 4: Axiomatization of TAll in the language $L_{B I}$.

Proof. As in Lemma 3 and 1, we simply list the set of necessary definitions:

$$
\begin{aligned}
I 44 J & \leftrightarrow \exists K(I 34 K \wedge K 34 J), \\
I 14 J & \leftrightarrow \exists K(I 34 \wedge \wedge K 03 J) \wedge \forall K(K 34 I \leftrightarrow K 34 J), \\
I 04 J & \leftrightarrow \exists K, L(K 34 I \wedge K 14 J \wedge I 34 L \wedge L 03 J), \\
I 24 J & \leftrightarrow \exists K, L(K 14 I \wedge K 34 J \wedge I 34 L \wedge L 03 J) .
\end{aligned}
$$

In all cases, the definitions are relatively straightforward.

Lemma 9. For each $r \in \mathcal{L}_{B I} \backslash \mathcal{L}_{L I},\{=, 34,03\} \rightarrow_{\text {All }} r$.

ProOF. All the definitions are the same with the ones presented in Lemma 3 and it is straightforward to check that all the proofs presented in Lemma 3 remain valid.

Lemma 7, 8, and 9 give us the following theorem.

Theorem 6. The set $\{=, 34,03\}$ is minimally $\mathcal{L}_{B I}$-complete on All.

Now, we present an axiomatization of $\Sigma_{B I}$, explicitly defined in Table 4 . Axioms (1) to (5) of Table 4 are the same as the ones presented in Table 2. However, in the general case we will need more axioms because we need to capture the semantics of more relations and the interplay between them. Note that we still make use of the relations $\sim$ and $<$ as defined in Section 3, and Lemma 4 still holds. We introduce a new relation, as follows:

$$
I \approx J \leftrightarrow(I=J \vee I 03 J \vee J 03 I)
$$


We use $\approx$ also as a shorthand for the formula which defines it. It is clear that $I \approx I$ and if $I \approx J$ then $J \approx I$, regardless of the semantics. Axiom (6) in Table 4 states that the relation $\approx$ is transitive and, hence, it is an equivalence relation. So, the relation $\sim$ relates intervals with the same starting point and $\approx$ relates intervals with the same ending point. Axiom (7) states that intervals with the same starting and ending point are equal. Axiom (8) imposes that if the starting point if $I$ is less than the starting point if $J$ then there is an interval starting together with $I$ and ending together with $J$. Axiom (9) imposes greatest lower bounds to endpoints of intervals and Axiom (10) states the idea that the starting points of intervals which have the same endpoint should not be incomparable. Finally Axiom (11) gives the relation between 34 and 03 .

Theorem 7. Let $\mathcal{I}$ be an $\{=, 34,03\}$-structure satisfying Axioms (1) to (11) in Table 4. Then, there is a future branching model of time $\mathcal{T}$, definably interpretable in $\mathcal{I}$, such that $\mathcal{I}$ is isomorphic to $\mathcal{I}(\mathcal{T})$.

Proof. Let $\mathcal{T}_{1}=\mathcal{I} / \sim$, be the set of equivalence classes of $\sim$ over $\mathcal{I}$. By Lemma $4,<$ induces a well-defined relation on $\mathcal{T}$ which we also denote by $<$. Note that $\left(\mathcal{T}_{1},<\right)$ is definably interpretable in $\mathcal{I}$ and it is a future branching model of time by Axioms (3), (4) and (5). Note also that, in the general case, $\mathcal{T}_{1}$ is not enough to capture all points, as we need also to capture possible greatest points of branches. To this end, we introduce the definable set $\mathcal{X}=\{I: \forall J \neg(I 34 J)\}$, which, intuitively, is the set of all cofinal intervals. Now let us define:

$$
\mathcal{T}=\mathcal{T}_{1} \biguplus(\mathcal{X} / \approx)
$$

We will equip $\mathcal{T}$ with an ordering, which extends the ordering $<$ on $\mathcal{T}_{1}$. Define a relation $R$ on $\mathcal{T}$ as follows:

$$
x R y \leftrightarrow\left\{\begin{array}{l}
x, y \in \mathcal{T}_{1} \text { and } x<y, \text { or } \\
x=(I / \sim) \in \mathcal{T}_{1}, y=(J / \approx) \in \mathcal{X} / \approx, \exists K(K \sim I \wedge K \approx J) .
\end{array}\right.
$$

Since both $\sim$ and $\approx$ are equivalence relations, the relation $R$ is well-defined, and, moreover, $(\mathcal{T}, R)$ is definably interpretable in $\mathcal{I}$. It is clear that $R$ is both irreflexive and antisymmetric. Now, suppose $x R y$ and $y R z$. Then, either $x, y, z \in \mathcal{T}_{1}$ or $x, y \in \mathcal{T}_{1}$ and $z \in \mathcal{X} / \approx$. In the former case, we immediately get $x R z$, since $<$ is a partial ordering on $\mathcal{T}_{1}$. Therefore, suppose we are in the latter case, with $x=I / \sim, y=J / \sim, z=K / \approx$. Then, 
$I<J$, and there is $L$ with $L \sim J$ and $L \approx K$. This gives us $I<L$, and by Axiom (8) there exists $M$ with $M \sim I$ and $M \approx L$. Since $\approx$ is an equivalence relation, $M \approx K$, together with $M \sim I$, gives us $x R z$. Hence, the relation $R$ is transitive, and so it is a partial order on $\mathcal{T}$ which extends the ordering $<$ on $\mathcal{T}_{1}$. From this point on, we also refer to $R$ as $<$. Axioms (9) and (10) ensure that $(\mathcal{T},<)$ is indeed a future branching model of time. Now, consider the set of intervals over $\mathcal{T}$ as a $\mathcal{L}$-structure where $\mathcal{L}=\{=, 34,03\}$ and the relations are interpreted in the natural way. Then, define $f: \mathcal{I} \rightarrow \mathcal{I}(\mathcal{T})$ as:

$$
f(I) \mapsto\left[I / \sim, I_{e}\right],
$$

where $I_{e}=I / \approx$ when $I \in \mathcal{X}$, and $I_{e}=J / \sim$ with $I 34 J$, otherwise. Note that in either case we have that $(I / \sim)<I_{e}$, and that $f(I)$ is an interval over $\mathcal{T}$. We claim that $f$ is an isomorphism of $\mathcal{L}$-structures. Suppose that $f(I)=f(J)$. Then, either both $I$ and $J$ are in $\mathcal{X}$ or they are both not in $\mathcal{X}$ as $I_{e}$ depends exactly on whether or not $I \in \mathcal{X}$. In the latter case we obtain $I=J$ from Axioms (1) and (2), as in the previous section, and in the former case we obtain $I=J$ from Axioms (1) and (7). So $f$ is one-to-one. Now, let $[x, y] \in \mathcal{I}(\mathcal{T})$. Then either $x, y \in \mathcal{T}_{1}$ or $x \in \mathcal{T}_{1}$ and $y \in \mathcal{X} / \approx$. In the former case we have that:

$$
x=(I / \sim)<y=(J / \sim),
$$

and, by the definition of $<$, there is $K \in \mathcal{I}$ such that $K \sim I$ and $K 34 J$. Therefore $f(K)=[x, y]$. In the latter case we have that:

$$
x=(I / \sim)<y=(J / \approx),
$$

so that there is $K$ with $K \sim I$ and $K \approx J$ and, hence, $f(K)=[x, y]$. This shows $f$ is onto. Finally, we need to show that $f$ respects the relations 34 and 03. Take $I, J \in \mathcal{I}$ such that I34J. Then $I \notin \mathcal{X}$, so $f(I)=[I / \sim, K / \sim]$ where $I 34 K$. Let $f(J)=\left[J / \sim, J_{e}\right]$. Then, by Axiom (1), we have $K \sim J$ and hence $f(I) 34 f(J)$. Next, take $I, J \in \mathcal{I}$ with $I 03 J$ : by Axiom (11), either both $I$ and $J$ are in $\mathcal{X}$ or they are both not in $\mathcal{X}$. In the former case we have that:

$$
f(I)=\left[I / \sim, K_{1} / \sim\right], \quad f(J)=\left[J / \sim, K_{2} / \sim\right],
$$

where $I 34 K_{1}$ and $J 34 K_{2}$. Using Axiom (11), again, we get $J 34 K_{1}$ as well. Therefore Axiom (1) gives us $K_{1} \sim K_{2}$. Using Axiom (11) one last time, we see $J<I$, and we get $f(I) 03 f(J)$. Now assume that both $I$ and $J$ are in $\mathcal{X}$. Then we have that:

$$
f(I)=[I / \sim, I / \approx], \quad f(J)=[J / \sim, J / \approx] .
$$


Since I03J, we immediately obtain $I \approx J$. Moreover, by Axiom (11), we have that $J<I$, so that $f(I) 03 f(J)$.

Theorem 8 (Representation Theorem). $\Sigma_{B I}$ is axiomatized by Axioms (1) to (11) in Tab. 4, together with the definitions of all relations in $\mathcal{L}_{B I}$ from $\{=, 34,03\}$.

Proof. It is clear that if $\mathcal{T}$ is a future branching model of time then (1) to (11) hold in $\mathcal{I}(\mathcal{T})$. Suppose that $\mathcal{I}$ is an $\mathcal{L}_{B I}$ structure satisfying (1) to (11), where each relation in $\mathcal{L}_{B I}$ is interpreted according to the definitions provided in Lemma 8 and Lemma 9. Then, by Theorem $7, \mathcal{I}$ is isomorphic to an interval structure $\mathcal{I}(\mathcal{T})$ and therefore $\mathcal{I} \models \Sigma_{B I}^{\text {TUnb }}$.

\section{Expressive Power over TAll}

We conclude our treatment of the first-order logic of branching structures by studying minimally complete and maximally incomplete subset of relations over TAll. The starting point of this analysis is the fact that $\{34,03,=\}$ is minimally complete over TAll.

Lemma 10. Each set listed in the right-hand side column of Tab. 5 is $\mathcal{L}_{B I^{-}}$ complete over TAll.

Proof. As in the previous results, let us proceed case-by-case. The case $S=\{34,03,=\}$ is already dealt with in Theorem 6 . Let $S=\{14,03,=\}$; by the completeness of $\{34,03,=\}$, it suffices to show that 34 is definable from $S$. To this end, as in Lemma 5 , it suffices to define $34 \cup 44$ from which one can immediately obtain 34 :

$$
I 34 \cup 44 J \leftrightarrow \exists K(I 14 K \wedge J 03 K) \wedge \forall K(K 03 I \rightarrow \neg K 14 J) .
$$

Now, let $S=\{24,66,03,=\}$. Thanks to the previous case, it suffices to show that 14 is definable from $S$ to prove that $S$ is complete. In the following definition, we introduce two unary relations $U(I)$ and $I S(I)$ to precisely identify those intervals that are, respectively, unit (that is $I=[a, b]$, and there exists no $c$ such that $a<c<b$ ), and initial segments (that is, $I=[a, b]$ 


\begin{tabular}{|l|l|}
\hline $\mathrm{MIS}\left(\mathcal{L}_{B I}\right) \mathrm{s}$ & $\operatorname{mcs}\left(\mathcal{L}_{B I}\right) \mathrm{s}$ \\
\hline $\mathcal{L}_{B I} \backslash\{16,=\}$ & $\{34,03,=\}$ \\
$\mathcal{L}_{B I} \backslash\{34,14,16,66\}$ & $\{14,03,=\}$ \\
$\mathcal{L}_{B I} \backslash\{03,14,16,66\}$ & $\{24,66,03,=\}$ \\
$\mathcal{L}_{B I} \backslash\{03,06\}$ & $\{04,66,03,=\}$ \\
$\mathcal{L}_{B I} \backslash\{34,03,66\}$ & $\{44,66,03,=\}$ \\
$\{14,=\} \cup\left(\mathcal{L}_{B I} \backslash \mathcal{L}_{L I}\right)$ & $\{34,03,16\}$ \\
$\{44,04,24,=\} \cup\left(\mathcal{L}_{B I} \backslash \mathcal{L}_{L I}\right)$ & $\{14,03,16\}$ \\
$\{03,=\} \cup\left(\mathcal{L}_{B I} \backslash \mathcal{L}_{L I}\right)$ & $\{24,03,16\}$ \\
& $\{44,03,16\}$ \\
& $\{04,03,16\}$ \\
& $\{34,66,06,=\}$ \\
& $\{34,14,06,=\}$ \\
& $\{14,24,66,06,=\}$ \\
& $\{14,04,66,06,=\}$ \\
& $\{14,44,66,06,=\}$ \\
& $\{34,16,06\}$ \\
& $\{14,24,66,16,06\}$ \\
& $\{14,04,66,16,06\}$ \\
& $\{14,44,66,16,06\}$ \\
\end{tabular}

Table 5: Maximally $\mathcal{L}_{B I}$-incomplete (left-hand side) and minimally $\mathcal{L}_{B I}$-complete sets (right-hand side) over TAll

and $a$ is the first point of the model). Now, we have:

$$
\begin{aligned}
I 14 J \leftrightarrow & (\neg I S(I) \wedge \neg(I 24 J) \wedge \forall K(I 03 K \rightarrow K 24 J)) \vee \\
& (I S(I) \wedge I S(J) \wedge \neg U(J) \wedge \\
& (U(I) \rightarrow \forall K(K 03 J \rightarrow \neg I 66 K)) \wedge \\
& (\neg U(I) \rightarrow \exists K(I 24 K \wedge K 03 J))) . \\
U(I) \leftrightarrow \leftrightarrow \forall J(\neg J 03 I) . & \forall \forall(\neg I 03 J) .
\end{aligned}
$$

Now, let $S=\{04,66,03,=\}$; to prove its completeness, we again define 14 in 
it, making use of the relations $U()$ and $I S()$ defined as above:

$$
\begin{aligned}
I 14 J \leftrightarrow & (\neg I S(J) \wedge \neg(I 04 J) \wedge \forall K(J 03 K \rightarrow I 04 K)) \vee \\
& (I S(I) \wedge I S(J) \wedge \neg U(J) \wedge \\
& (U(I) \rightarrow \forall K(K 03 J \rightarrow \neg I 66 K)) \wedge \\
& (\neg U(I) \rightarrow \exists K(K 03 I \wedge K 04 J))) .
\end{aligned}
$$

Consider, now, $S=\{03,44,66,=\}$. To prove its completeness, we define 34 in it, and the corresponding definition is:

$$
\begin{aligned}
I 34 J \leftrightarrow & (\forall K(\neg S T(J, K) \vee \neg F I(I, K)) \wedge \neg I 44 J) \wedge \\
& \forall K(K 03 J \rightarrow I 44 K) \wedge \\
& \forall K(I 03 K \rightarrow \neg(K 66 J)) \wedge \\
& (\neg I S(J) \wedge(\neg I S(I) \rightarrow \exists K(K 44 J \wedge \neg(K 44 I)))) \wedge \\
& \forall K(K 03 I \rightarrow \neg(K 66 J)) \wedge \\
& \forall K(K 03 J \rightarrow \neg(K 66 I)) . \\
S T(I, J) \leftrightarrow & (\exists K(I 03 K) \leftrightarrow \exists K(J 03 K)) \wedge \forall K(K 44 I \leftrightarrow K 44 J) . \\
F I(I, J) \leftrightarrow & (I 03 J \vee J 03 I \vee I=J) .
\end{aligned}
$$

Let, now, $S=\{34,03,16\}$. In this case, it suffices to show that $=$ is definable:

$$
\begin{aligned}
I=J \leftrightarrow & \neg(I 16 J) \wedge \\
& \forall K(K 34 I \leftrightarrow K 34 J) \wedge \forall K(I 34 K \leftrightarrow J 34 J) \wedge \\
& \forall K, L((K 03 I \wedge L 03 J) \rightarrow \neg K 16 L) .
\end{aligned}
$$

For the next four cases we first observe that the relation $6 r$, introduced immediately before Lemma 2, is definable using 03 and 16 only:

$$
\begin{aligned}
I 6 r J \leftrightarrow & I 16 J \vee \exists K(K 03 J \wedge I 16 K) \vee \exists K(J 03 K \wedge I 16 K) \vee \\
& \exists K(K 03 I \wedge J 16 K) \vee \exists K(I 03 K \wedge J 16 K) \vee \\
& \exists K, L(K 03 I \wedge L 03 J \wedge K 16 L) \vee \exists K, L(I 03 K \wedge L 03 J \wedge K 16 L) \vee \\
& \exists K, L(K 03 I \wedge J 03 L \wedge K 16 L) \vee \exists K, L(I 03 K \wedge J 03 L \wedge K 16 L) .
\end{aligned}
$$

It is straightforward to check that $I$ and $J$ are related through a branching relation if and only if the right hand side of the equivalence is satisfied. Now let $S=\{14,03,16\}$; defining $34 \cup 44$ can be accomplished as follows:

$$
I 34 \cup 44 J \leftrightarrow \exists K(I 14 K \wedge J 14 K) \wedge \neg \exists K(K 03 I \wedge K 14 J) \wedge \neg(I 6 r J) .
$$

It is immediate to see that the above definition imposes $I$ and $J$ to be linearly related, and, then, to be related by $34 \cup 44$ as we wanted. Consider now 
$S=\{24,03,16\} ;$ a similar, but slightly more elaborate argument works:

$$
\begin{aligned}
I 34 \cup 44 J \leftrightarrow & \neg(I 24 J) \wedge \neg(J 24 I) \wedge \neg(I 03 J) \wedge \neg(J 03 I) \wedge \\
& \exists K(J 03 K \wedge \neg(I 03 K)) \wedge \forall K(I 03 K \rightarrow \neg(K 24 J)) \wedge \\
& \forall K, L((J 03 K \wedge L 03 I) \rightarrow \neg(K 24 L)) \wedge \\
& \forall K(J 03 K \rightarrow \neg(K 24 I)) \wedge \\
& \forall K, L((J 03 K \wedge I 03 L) \rightarrow \neg(K 24 L)) \wedge \neg(I 6 r J) .
\end{aligned}
$$

As for the case $S=\{44,03,16\}$, we can immediately use the definitions of $S T()$ and $F I()$ from a previous case to obtain 34, which is enough to prove that $S$ is complete thanks to the completeness of $\{34,03,16\}$ :

$$
I 34 J \leftrightarrow(\forall K(\neg S T(J, K) \vee \neg F I(I, K)) \wedge \neg I 44 J) \wedge \neg(I 6 r J) .
$$

If $S=\{04,03,16\}$ we can apply a similar schema to reduce ourselves to a linear case, and define, in this case, the relation 24 , which is enough to get the completeness thanks to the already proved completeness of $\{24,03,16\}$ :

$$
\begin{aligned}
I 24 J \leftrightarrow & \exists K(J 03 K \wedge \neg(I 04 K) \wedge \exists L(L 04 K \wedge L 03 I)) \wedge \\
& \exists K(K 03 I \wedge \forall L(J 04 L \rightarrow K 04 L)) \wedge \neg(I 6 r J) .
\end{aligned}
$$

Keeping in mind that the last conjunct makes sure that $I$ and $J$ are linearly related, the correctness of this definition becomes straightforward. Let $S=$ $\{34,66,06,=\}$; we have previously shown that $\{34,03,=\}$ is complete, so it suffices to show that 03 is definable from $S$ :

$$
\begin{aligned}
I 03 J \leftrightarrow & \exists K \forall L(K 34 I \wedge(L 34 K \leftrightarrow L 34 J)) \wedge \\
& \forall K(I 34 K \leftrightarrow J 34 K) \wedge \forall K(K 06 J \rightarrow \neg K 34 I) \wedge \\
& \forall K(K 06 I \rightarrow K 06 J) \wedge \neg(I 06 J) \wedge \neg(J 66 I) .
\end{aligned}
$$

Let $S=\{34,14,06,=\}$. Again, it suffices to show that 03 is definable since $\{34,03,=\}$ is complete:

$$
\begin{aligned}
I 03 J \leftrightarrow & \exists K(K 34 I \wedge K 14 J) \wedge \forall K(I 34 K \leftrightarrow J 34 K) \wedge \\
& \forall K(K 06 I \rightarrow K 06 J) \wedge \neg(I 06 J) .
\end{aligned}
$$

Now, let $S=\{14,24,66,06,=\}$. To prove the completeness of this set, we first redefine the unary relation $U()$ (defining it via 14 instead of 03 ), and then we substitute the relation $I S($ ) with its symmetric version $F S($ ) (final segment); we have that $F S(I)$ holds if and only if the ending point of $I$ 
is a maximal point of $\mathcal{T}$. Now, we define 03, which suffices to prove the completeness of $S$ since $\{14,03,=\}$ is complete:

$$
\begin{aligned}
I 03 J \leftrightarrow \leftrightarrow & (\neg F S(I) \wedge \forall K(I 14 K \rightarrow J 24 K) \wedge \neg J 24 I) \vee \\
& (F S(I) \wedge F S(J) \wedge \neg U(J) \wedge(U(I) \rightarrow(\neg J 66 I \wedge \neg I 06 J)) \wedge \\
& (\neg U(I) \rightarrow(\exists K(K 14 J \wedge K 24 I)) \wedge \forall K(K 06 I \rightarrow K 06 J))), \\
U(I) \leftrightarrow \leftrightarrow \forall J(\neg J 14 I), & F S(I) \leftrightarrow \forall J(\neg I 14 J) .
\end{aligned}
$$

If $S=\{14,04,66,06,=\}$, we use the unary relations $U()$ and $F S()$ to define 03, which suffices to show that $S$ is complete:

$$
\begin{aligned}
I 03 J \leftrightarrow & (\neg F S(I) \wedge \forall K(J 14 K \rightarrow I 04 K) \wedge \neg I 04 J) \vee \\
& (F S(I) \wedge F S(J) \wedge \neg U(J) \wedge(U(I) \rightarrow(\neg J 66 I \wedge \neg I 06 J)) \wedge \\
& (\neg U(I) \rightarrow(\exists K(K 14 I \wedge K 04 J) \wedge \forall K(K 06 I \rightarrow K 06 J)))) .
\end{aligned}
$$

Let $S=\{14,44,66,06,=\}$. We define, as other cases before, the relation $34 \cup 44$. The auxiliary relations $S T()$ and $F I()$, defined using 14 instead of 44 , will be used here; observe that their semantics is not changed. To define $34 \cup 44$, we use:

$$
\begin{aligned}
I 34 \cup 44 J \leftrightarrow & \forall K(\neg S T(J, K) \vee \neg F I(I, K)) \wedge \exists K(I 14 K) \wedge \\
& \left(\bigwedge_{r \in\{06,66,=\}}(\neg I r J \wedge \neg J r I)\right) \wedge \\
& \forall K(I 66 K \rightarrow J 66 K) \wedge \forall K(J 66 K \rightarrow I 44 K) \wedge \\
& \forall K(K 06 J \rightarrow I 44 K) \wedge \forall K(K 06 I \rightarrow K 66 J) . \\
S T(I, J) \leftrightarrow \leftrightarrow & (I 14 J \vee J 14 I \vee I=J) . \\
F I(I, J) \leftrightarrow \leftrightarrow & (\exists K(I 14 K) \leftrightarrow \exists K(J 14 K)) \wedge \\
& \forall K(I 44 K \leftrightarrow J 44 K) .
\end{aligned}
$$

Continuing, let $S=\{34,16,06\}$. We show that $=$ and 66 are definable from $S$, which suffices to show completeness of $S$, since $\{34,66,06,=\}$ was previously shown to be complete:

$$
\begin{aligned}
& I 66 J \leftrightarrow \exists K(K 16 I \wedge K 34 J) . \\
& I=J \leftrightarrow \quad \forall K(K 34 I \leftrightarrow K 34 J) \wedge \forall K(I 34 K \leftrightarrow J 34 K) \wedge \\
& \neg I 16 J \wedge \forall K(K 06 I \rightarrow K 06 J) .
\end{aligned}
$$

Finally, as for the cases $S=\{14,24,66,16,06\}$ and $S^{\prime}=\{14,04,66,16,06\}$, observe that we were able to define 03 from $\{14,24,66,06,=\}$ without using equality. Therefore 03 is definable from $S$, and $S$ is complete. Likewise, we 
are also able to define 03 from $\{14,04,66,06,=\}$ without using equality, so that $S^{\prime}$ defines 03 and it is also complete. Let $S=\{14,44,66,16,06\}$. It suffices to show that $=$ is definable since $\{14,44,66,06,=\}$ is already known to be complete:

$$
\begin{aligned}
I=J \leftrightarrow & \forall K(I 14 K \leftrightarrow J 14 K) \wedge \forall K(K 14 I \leftrightarrow K 14 J) \wedge \\
& \forall K(K 66 I \leftrightarrow K 66 J) \wedge \forall K(K 06 I \leftrightarrow K 06 J) \wedge \\
& (\neg I 16 J \wedge \neg I 66 J \wedge \neg I 06 J) .
\end{aligned}
$$

This concludes the proof, having analyzed all sets listed in the left-hand side of Tab. 5.

Lemma 11. Each set listed in the left-hand side column of Tab. 5 is $\mathcal{L}_{B I^{-}}$ incomplete over TAll.

Proof. The incompleteness of the first three cases is implicit in the proof of Lemma 7. For example, let $S=\mathcal{L}_{B I} \backslash\{16,=\}$, let $\mathcal{T}=\{a, b, c\}$ with $a<b, a<c$ and $b \| c$, and let $f: \mathcal{I}(\mathcal{T}) \rightarrow \mathcal{I}(\mathcal{T})$ be the map which sends both $[a, b]$ and $[a, c]$ to $[a, b]$ (that is, the first construction presented in the proof of Lemma 7). There are no intervals over $\mathcal{T}$ which are related via any relation in $S$, implying that these are all preserved, but, clearly, $=$ and 16 are not preserved. So $S=\mathcal{L}_{B I} \backslash\{16,=\}$, the first one in the list, is incomplete. Similar arguments, based on the proof of Lemma 7, allow us to prove the incompleteness of the second and third set in the list. Now, let $S=\mathcal{L}_{B I} \backslash\{03,06\}$. Take $\mathcal{T}=\{a, b\} \cup\left\{c_{i}: i \in \mathbb{Z}\right\}$ which is ordered by $a<b<c_{i}$, with $c_{i} \| c_{j}$ for all distinct $i, j \in \mathbb{Z}$; this is to say that starting with the interval $[a, b]$, the $c_{i}$ s fan out, and they are all incomparable to each other. Let $f: \mathcal{I}(\mathcal{T}) \rightarrow \mathcal{I}(\mathcal{T})$ be the map which sends $\left[b, c_{i}\right]$ to $\left[b, c_{i+1}\right]$ for $i \in \mathbb{Z}$ and fixes all other intervals. Once again, it is easy to see that all relations in $S$ are preserved; however, we have $\left[b, c_{0}\right] 03\left[a, c_{0}\right]$ and $\neg\left[b, c_{1}\right] 03\left[a, c_{0}\right]$. Hence $S$ is incomplete. Next, let $S=\mathcal{L}_{B I} \backslash\{34,03,66\}$. Let $\mathcal{T}=\{a\} \cup\left\{b_{i}: i \in\right.$ $\mathbb{Z}\} \cup\left\{c_{i}: i \in \mathbb{Z}\right\}$ be the future branching model of time, which is ordered by $a<b_{i}<c_{i}$, with $b_{i}|| b_{j}$ for all distinct $i, j \in \mathbb{Z}$; this is to say that, similarly to the previous model, starting at $a$, the branches $b_{i}<c_{i}$ fan out and they are all incomparable to each other. Let $f: \mathcal{I}(\mathcal{T}) \rightarrow \mathcal{I}(\mathcal{T})$ be the map which sends $\left[b_{i}, c_{i}\right]$ to $\left[b_{i+1}, c_{i+1}\right]$ and fixes all other intervals. Then $f$ preserves all relations in $S$; however $\left[a, b_{0}\right] 34\left[b_{0}, c_{0}\right]$ and $\neg\left[a, b_{0}\right] 34\left[b_{1}, c_{1}\right]$, so that $S$ is incomplete. As far as the sets $S=\{03,=\} \cup\left(\mathcal{L}_{B I} \backslash \mathcal{L}_{L I}\right)$ and $S=\{14,=\} \cup\left(\mathcal{L}_{B I} \backslash \mathcal{L}_{L I}\right)$ are concerned, it suffices to recall that incompleteness is preserved from smaller 
to wider classes of models, and that these two sets have been proven to be incomplete in the unbounded case in Lemma 6, implying that they must be incomplete in the general case as well. So, it remains to be shown that $S=$ $\{44,04,24,=\} \cup\left(\mathcal{L}_{B I} \backslash \mathcal{L}_{L I}\right)$ is also incomplete. To this end, let $\mathcal{T}=\{a, b, c\}$ ordered by $a<b<c$, and define $f: \mathcal{I}(\mathcal{T}) \rightarrow \mathcal{I}(\mathcal{T})$ in such a way to send $[a, c]$ to itself and exchange the other two intervals: all non-linear relations are preserved in a trivial way, and the same holds for the relations 44, 24,04, and $=$. But, obviously, 34 is broken, proving the incompleteness of this set, completing the proof.

Theorem 9. Tab. 5 contains all minimally $\mathcal{L}_{B I}$-complete and maximally $\mathcal{L}_{B I}$-incomplete subsets of $\mathcal{L}_{B I}$ over TAll.

Proof. Observe that in Tab. 5 every subset of a complete set is contained in an incomplete set, and every superset of an incomplete set contains a complete set. Moreover, it is easy to see that each given subset of $\mathcal{L}_{B I}$ is either (not necessarily strictly) contained in one of those listed in the lefthand side column or it (not necessarily strictly) contains one of those listed in the right-hand side column. Therefore, the left-hand side column of Table 5 is the list of all maximally incomplete sets and the rightmost column is the list of all minimally complete sets over TAll.

\section{Conclusions}

In this paper we properly extended Allen's theory of time from linear flows of time to branching tree-like structures. We did so in both the unbounded case, which turned out to be fairly simple, and in which Allen's relation meets remains complete, and in the general case, where we proved that the smallest complete set of relations has cardinality three. In both the unbounded and the general case we provided sound and complete axiomatic systems in the language of branching relations. These axioms are indeed natural generalizations of the corresponding axioms from the linear case to the branching case, and we showed that an abstract interval structure satisfying these axioms is indeed a concrete interval structure based on a branching model of time which is interpretable in the abstract interval structure. Therefore the concept of a representation theorem is generalized in full strength to the case of branching interval structures. Then, we systematically studied minimal completeness and maximal incompleteness for 
a set of branching relations, again, in the unbounded and the general case; we proved that there are precisely four maximally incomplete and eight minimally complete sets in the former case, while in the latter case these are, respectively, eight and nineteen. Our work differs from [3], which is focused, unlike the present paper, on more practical complexity and algorithmic problems concerning the interval algebra that emerges from branching relations, and leaves unexplored the theoretical basis; as a matter of fact the problem of studying minimally complete and maximally incomplete subsets of (linear and) branching relations for tree-like lattices was left open in [3] (Remark 5). It is a mistake to think that interval algebras are a closed topic and that no more interesting problems stand. To give a short account, and point to some possible natural research directions, consider what follows. Thanks to all the work that followed the introduction of Allen's Interval Algebra, which includes Ligozat, Condotta, and Balbiani's papers [7, 23, 24], studying interval algebras in the linear case can be considered as a closed subject; but the pure interval branching case [3], as well as the point-interval linear case $[2,25]$ have not been studied in their full extent, and the study of the branching case that include intervals and points is, to the best of out knowledge, limited to [4]. While on the short run we plan to consider all interesting classes of tree-like lattices other than the two considered here, and a similar study for the linear case enriched with points is the object of a forthcoming paper, in the long run we will also consider a systematic treatment of the algebras the emerge in the (linear and) branching case with and without points, also in the decidability/complexity point of view; while it is well known that in the linear case deciding satisfiability of a set of constraints is NP-complete, and the entire spectrum of sub-algebras has been studied, this problem has been approached in the branching case only to a very limited extent.

\section{Acknowledgements}

The authors acknowledge the support from the Italian INdAM-GNCS project Logics and Automata for Interval Model Checking (G. Sciavicco).

\section{References}

[1] J. Allen, Maintaining knowledge about temporal intervals, Communications of the ACM 26 (11) (1983) 832-843. 
[2] W. Conradie, S. Durhan, G. Sciavicco, An integrated first-order theory of points and intervals: Expressive power in the class of all linear orders, in: Proc. of the 19th International Symposium on Temporal Representation and Reasoning (TIME), IEEE, 2012, pp. $47-51$.

[3] M. Ragni, S. Wölfl, Branching Allen, in: Proc. of the 4th International Conference on Spatial Cognition, Vol. 3343 of Lecture Notes in Computer Science, Springer, 2004, pp. 323-343.

[4] A. Reich, Intervals, points, and branching time, in: Proc. of the 9th International Symposium on Temporal Representation and Reasoning (TIME), IEEE, 1994, pp. 121-133.

[5] P. Ladkin, Models of axioms for time intervals, in: Proc. of the 6th National Conference on Artificial Intelligence, Morgan Kaufmann, 1987, pp. 234-239.

[6] A. Galton, Note on a lemma of Ladkin, Journal of Logic and Computation 6 (1) (1996) 1-4.

[7] G. Ligozat, On generalized interval calculi, in: Proc. of the 9th National Conference on Artificial Intelligence (AAAI), 1991, pp. 234-240.

[8] A. Molinari, A. Montanari, A. Murano, G. Perelli, A. Peron, Checking interval properties of computations, Acta Informatica 56 (6-8) (2016) $587-619$.

[9] J. van Benthem, The Logic of Time (2nd Edition), Kluwer Academic Press, 1991.

[10] J. Allen, P. Hayes, A common-sense theory of time, in: Proc. of the 9th International Joint Conference on Artificial Intelligence, Morgan Kaufmann, 1985, pp. 528-531.

[11] P. Ladkin, The Logic of Time Representation, Ph.D. thesis, University of California, Berkeley (1978).

[12] Y. Venema, A modal logic for chopping intervals, Journal of Logic and Computation 1 (4) (1991) 453-476. 
[13] V. Goranko, A. Montanari, G. Sciavicco, Propositional interval neighborhood temporal logics, Journal of Universal computer science 9 (9) (2003) 1137-1167.

[14] A. Bochman, Concerted instant-interval temporal semantics I: Temporal ontologies, Notre Dame Journal of Formal Logic 31 (3) (1990) 403-414.

[15] C. J. Coetzee, Representation theorems for classes of interval structures, Master's thesis, Department of Mathematics, University of Johannesburg (2009).

[16] R. Thomason, Combination of tense and modality, in: D. Gabbay, F. Guenthner (Eds.), Handbook of Philosophical Logic, Vol. II, 1984, pp. 135-165.

[17] R. Alur, T. Henzinger, O. Kupferman, Alternating-time temporal logic, Journal of the ACM 49 (5) (2002) 672-713.

[18] E. A. Emerson, Temporal and modal logic, in: Handbook of Theoretical Computer Science, Volume B: Formal Models and Sematics (B), MIT Press, 1990, pp. 995-1072.

[19] D. Bresolin, A. Montanari, P. Sala, An optimal tableau for right propositional neighborhood logic over trees, in: Proc. of the 15th International Symposium on Temporal Representation and Reasoning (TIME), IEEE, 2008, pp. 110-117.

[20] F. van Kutshera, Sebastian's strolls, Grazer Philosophisce Studien (45) (1993) 75-88.

[21] J. Allen, P. J. Hayes, Short time periods, in: Proc. of the 10th International Joint Conference on Artificial Intelligence, 1987, pp. 981-983.

[22] W. Conradie, G. Sciavicco, On the expressive power of first order-logic extended with allen's relations in the strict case, in: Proc. of the 2011 Conferencia Española para la Inteligencia Artificial (CAEPIA), Vol. 7023 of Lecture Notes in Computer Science, Springer, 2011, pp. 173182.

[23] J. Condotta, Tractable sets of the generalized interval algebra, in: Proc. of the 14th European Conference on Artificial Intelligence (ECAI), 2000, pp. $78-82$. 
[24] P. Balbiani, J. Condotta, . Ligozat, Reasoning about generalized intervals: Horn representability and tractability, in: Proc. of the 7th International Workshop on Temporal Representation and Reasoning (TIME), 2000, pp. 23-29.

[25] I. Navarrete, A. Sattar, R. Marín, Deciding consistency of a pointduration network with metric constraints, in: Proc of the 10th International Symposium on Temporal Representation and Reasoning (TIME), 2003, pp. 147-154. 